\title{
Investigation into the association between NLRP3 gene polymorphisms and susceptibility to type 2 diabetes mellitus
}

\author{
S. Wang ${ }^{1}$, F. Fang ${ }^{2}$, W.B. Jin ${ }^{1}$, X. Wang ${ }^{3}$ and X.S. Zheng ${ }^{4}$ \\ 1Department of Endocrinology, Nanyang City Center Hospital, Nanyang, China \\ 2Department of Computed Tomography, Nanyang City Center Hospital, Nanyang, \\ China \\ ${ }^{3}$ Department of Nursing, Nanyang City Center Hospital, Nanyang, China \\ ${ }^{4}$ Intensive Care Unit, Nanyang City Center Hospital, Nanyang, China \\ Corresponding author: X.S. Zheng \\ E-mail: zhengxss@163.com \\ Genet. Mol. Res. 14 (4): 17447-17452 (2015) \\ Received August 24, 2015 \\ Accepted October 7, 2015 \\ Published December 21, 2015 \\ DOI http://dx.doi.org/10.4238/2015.December.21.15
}

ABSTRACT. We conducted a case-control study to investigate the role of three common polymorphisms (rs10754558, rs7512998, and rs12137901) of the gene NLR family, pyrin domain containing 3 (NLRP3) in the development of type 2 diabetes mellitus (T2DM). Between May 2013 and May 2014, 385 patients with T2DM and 401 control subjects were enrolled in our study. Genotyping of the three NLRP3 polymorphisms of interest was performed by polymerase chain reaction-restriction fragment length polymorphism. Unconditional logistic regression analyses showed that individuals carrying GG and GC+GG rs 10754558 genotypes were at significantly increased risk of T2DM, with adjusted odds ratios (and 95\% confidence intervals) of 1.81 (1.16-2.83) and 1.40 (1.04-1.88), respectively. In conclusion, we propose that the NLRP3 rs 10754558 polymorphism contributes to the development of T2DM, but that rs7512998 and rs12137901 variants are not associated with susceptibility to this disease.

Key words: NLRP3 polymorphism; Type 2 diabetes; Chinese population 


\section{INTRODUCTION}

Type 2 diabetes mellitus (T2DM) is a common, complex, and chronic disease, and its associated complications cause high morbidity, mortality, and disability worldwide. It is reported that approximately 92.4 million adult T2DM cases and 148.2 million individuals with prediabetes in China in 2010 (Yang et al., 2010). The development of T2DM is a complex, multistep, and multifactorial process, involving various environmental and genetic factors. Previous studies have reported that hyperglycemia, hypertension, dyslipidemia, and increased blood sugar levels contribute to the development of this disease (Ahmadi et al., 2014; Chen, Pei, et al., 2015). However, not all individuals exposed to similar risk factors develop T2DM, suggesting that genetic variations might influence susceptibility. Several previous studies have reported that gene polymorphisms play an important role in T2DM risk, including variations in MTHFR, TCF7L2, G1057D, TNF- $\alpha$, and IGF2R (Chanprasertyothin et al., 2015; Chen et al., 2015; Golshani et al., 2015; Jiang et al., 2015; Nanfa et al., 2015).

The NLRP3 gene is located in 1q44, and is reported to be associated with many immunemediated inflammatory diseases, such as rheumatoid arthritis, Crohn's disease, celiac disease, and type 1 diabetes (Kastbom et al., 2008; Villani et al., 2009; Pontillo et al., 2010). However, only one study has documented an association between NLRP3 polymorphism and T2DM (Zheng et al., 2013). Therefore, we conducted a case-control study to investigate the role of three common NLRP3 polymorphisms (rs10754558, rs7512998, and rs12137901) in the development of T2DM.

\section{MATERIAL AND METHODS}

\section{Patients}

T2DM patients were recruited from Nanyang City Center Hospital between May 2013 and May 2014. Individuals were diagnosed according to the revised American Diabetes Association criteria (Expert Committee on the Diagnosis and Classification of Diabetes Mellitus, 2003). A total of 426 T2DM patients were identified, 385 of whom agreed to be included in our study, for a participation rate of $90.38 \%$. Patients who had suffered acute or chronic inflammatory diseases, infections, cancer, or end-stage liver or kidney diseases were excluded from our study.

In total, 426 healthy control subjects attending health examination clinics at the study hospital were approached. Individuals with a history of diabetes, cancer, end-stage liver or kidney disease, or other metabolic disorders were excluded from our study. Finally, 401 control subjects were enrolled, resulting in a participation rate of $94.13 \%$.

Demographic characteristics were determined using a standardized questionnaire, and included age, gender, and body mass index (BMI). Clinical characteristics were collected from medical records, and included blood glucose, insulin, and glycosylated hemoglobin $\left(\mathrm{HbA}_{1 \mathrm{c}}\right)$ levels, as well as a lipid profile consisting of serum total cholesterol (TC), triglycerides (TGs), and high- and lowdensity lipoprotein cholesterol (HDL-C and LDL-C, respectively) measurements. A signed informed consent form was obtained from every participant before their inclusion in the study. This protocol was approved by the Clinical Research Ethics Committee of the Nanyang City Center Hospital.

\section{Genotyping analysis}

Peripheral blood samples were collected in ethylenediaminetetraacetic acid-coated tubes and stored at $-20^{\circ} \mathrm{C}$ until needed. DNA was isolated from these samples by salt extraction. Geno- 
typing of the NLRP3 sequence variants rs10754558, rs7512998, and rs12137901 was performed by polymerase chain reaction (PCR)-restriction fragment length polymorphism. The primers used were as follows: for rs10754558, 5'-CCG GGT TCA ATC AAT TCT CCT-3' (forward) and 5'-CGG GCG TGG AGG TGC AAT G-3' (reverse); for rs7512998, 5'-CCG GGC ATG GTG GCT CA-3' (forward) and 5'-CCC CCG GCT GCA CAC TG-3' (reverse); for rs12137901, 5'-GGG CGT GCA GTC CTG AAG T-3' (forward) and 5'-CCC CAG TCG CTT ACC TTT CTT C-3' (reverse). The PCR was performed using the following conditions: an initial DNA denaturation step at $94^{\circ} \mathrm{C}$ for $5 \mathrm{~min}$, then 30 cycles of denaturation at $94^{\circ} \mathrm{C}$ for $1 \mathrm{~min}$, annealing at $55^{\circ} \mathrm{C}$ for $1 \mathrm{~min}$, and extension at $72^{\circ} \mathrm{C}$ for $2 \mathrm{~min}$, followed by a final extension step at $72^{\circ} \mathrm{C}$ for $5 \mathrm{~min}$.

\section{Statistical analysis}

Statistically significant differences between cases and controls regarding demographic characteristics were assessed by the chi-square test, and the distribution of genotypes in the control group was tested for deviation from Hardy-Weinberg equilibrium. Associations between each of the NLRP3 polymorphisms and T2DM occurrence were analyzed by calculating odds ratios (ORs), 95\% confidence intervals $(95 \% \mathrm{Cls})$, and their corresponding $\mathrm{P}$ values. Statistical analysis was conducted using SPSS 17.0 (SPSS Inc., Chicago, IL, USA). P values lower than 0.05 were considered to be statistically significant.

\section{RESULTS}

The demographic and clinical characteristics of the T2DM patients and healthy controls are shown in Table 1. As expected, no significant differences were detected between the two groups in terms of gender and age $(P>0.05)$. In comparison to the control group, T2DM patients were more likely to have a higher BMI, and elevated levels of fasting glucose, fasting insulin, TC, TGs, HDL-C, and LDL-C (P < 0.05).

Table 1. Demographic and clinical characteristics of type 2 diabetes mellitus patients and control subjects.

\begin{tabular}{|c|c|c|c|c|c|c|}
\hline Characteristic & Patients $(\mathrm{N}=385)$ & $\%$ & Controls $(N=401)$ & $\%$ & Chi-square & $P$ value \\
\hline \multicolumn{7}{|l|}{ Age (years) } \\
\hline$<50$ & 213 & 55.32 & 211 & 52.62 & & \\
\hline$\geq 50$ & 172 & 44.68 & 190 & 47.38 & 0.58 & 0.45 \\
\hline \multicolumn{7}{|l|}{ Gender } \\
\hline Female & 147 & 38.18 & 175 & 43.64 & & \\
\hline Male & 238 & 61.82 & 226 & 56.36 & 2.42 & 0.12 \\
\hline \multicolumn{7}{|l|}{ BMI } \\
\hline$<24$ & 179 & 46.49 & 220 & 54.86 & & \\
\hline$\geq 24$ & 206 & 53.51 & 181 & 45.14 & 5.34 & 0.02 \\
\hline Fasting glucose (mM) & & $8.50 \pm 2.7$ & & $4.60 \pm 1.20$ & 26.34 & $<0.001$ \\
\hline Fasting insulin (mM) & & $58.30 \pm 15.6$ & & $52.40 \pm 14.90$ & 5.42 & $<0.001$ \\
\hline $\mathrm{HbA1c}$ & & $8.50 \pm 1.02$ & & $5.10 \pm 0.77$ & 52.88 & $<0.001$ \\
\hline $\mathrm{TC}(\mathrm{mg} / \mathrm{dL})$ & & $190.50 \pm 16.30$ & & $155.00 \pm 10.60$ & 36.34 & $<0.001$ \\
\hline TGs (mg/dL) & & $175.30 \pm 15.80$ & & $133.60 \pm 17.20$ & 35.36 & $<0.001$ \\
\hline $\mathrm{HDL}-\mathrm{C}(\mathrm{mg} / \mathrm{dL})$ & & $48.20 \pm 12.20$ & & $46.50 \pm 11.90$ & 1.98 & 0.02 \\
\hline LDL-C (mg/dL) & & $136.30 \pm 11.40$ & & $117.50 \pm 8.70$ & 26.05 & $<0.001$ \\
\hline
\end{tabular}

$\mathrm{BMI}=$ body mass index; HbA1c = glycosylated hemoglobin; $\mathrm{TC}=$ total cholesterol; $\mathrm{TGs}=$ triglycerides; $\mathrm{HDL}-\mathrm{C}=$ highdensity lipoprotein cholesterol; LDL-C = low-density lipoprotein cholesterol. 
The distributions of NLRP3 rs10754558, rs7512998, and rs12137901 polymorphisms are shown in Table 2. The genotype frequencies of rs10754558 and rs7512998 in the control group were found to be consistent with Hardy-Weinberg equilibrium (with $P$ values of 0.21 and 0.07 , respectively), while that of $r s 12137901$ was not $(P=0.04)$. Unconditional logistic regression analyses showed that individuals carrying the rs 10754558 GG and GC+GG genotypes were at significantly increased risk of T2DM, with adjusted ORs (and 95\% Cls) of 1.81 (1.16-2.83) and 1.40 (1.04-1.88), respectively.

Further analysis was conducted to identify any association between the rs 10754558 polymorphism and the demographic and clinical characteristics related to T2MD considered in this investigation.

Table 2. Association between NLRP3 polymorphisms and risk of type 2 diabetes mellitus.

\begin{tabular}{|c|c|c|c|c|c|c|c|}
\hline Polymorphism & Patients & $\%$ & Controls & $\%$ & $P$ value for HWE in control group & OR $(95 \% \mathrm{Cl})^{1}$ & $P$ value \\
\hline \multicolumn{8}{|l|}{ rs10754558 } \\
\hline $\mathrm{CC}$ & 147 & 38.18 & 186 & 46.38 & & 1.0 (Ref.) & - \\
\hline CG & 168 & 43.64 & 166 & 41.40 & & $1.30(0.95-1.78)$ & 0.09 \\
\hline GG & 70 & 18.18 & 49 & 12.22 & 0.21 & $1.81(1.16-2.83)$ & 0.005 \\
\hline$C G+G G$ & 238 & 61.82 & 215 & 53.62 & & $1.40(1.04-1.88)$ & 0.02 \\
\hline \multicolumn{8}{|l|}{ rs7512998 } \\
\hline $\mathrm{TT}$ & 170 & 44.16 & 189 & 47.13 & & 1.0 (Ref.) & - \\
\hline $\mathrm{TC}$ & 160 & 41.56 & 161 & 40.15 & & $1.10(0.81-1.51)$ & 0.52 \\
\hline $\mathrm{CC}$ & 55 & 14.28 & 51 & 12.72 & 0.07 & $1.20(0.76-1.89)$ & 0.41 \\
\hline $\mathrm{TC}+\mathrm{CC}$ & 215 & 55.84 & 212 & 52.87 & & $1.13(0.84-1.51)$ & 0.40 \\
\hline \multicolumn{8}{|l|}{ rs12137901 } \\
\hline $\mathrm{CC}$ & 179 & 46.49 & 197 & 49.13 & & 1.0 (Ref.) & - \\
\hline $\mathrm{CT}$ & 155 & 40.26 & 155 & 38.65 & & $1.10(0.81-1.50)$ & 0.53 \\
\hline $\mathrm{TT}$ & 51 & 13.25 & 49 & 12.22 & 0.04 & $1.15(0.72-1.83)$ & 0.55 \\
\hline $\mathrm{CT}+\mathrm{TT}$ & 206 & 53.51 & 204 & 50.87 & & $1.11(0.83-1.49)$ & 0.46 \\
\hline
\end{tabular}

${ }^{1}$ Adjusted for age, gender, body mass index, fasting glucose, fasting insulin, glycosylated hemoglobin, total cholesterol, triglycerides, and high- and low-density lipoprotein cholesterol. HWE = Hardy-Weinberg equilibrium; OR = odds ratio; $\mathrm{Cl}=$ confidence interval; Ref. = reference.

\section{DISCUSSION}

$N L R P 3$ has been reported to be involved in the recognition of several pathogen-associated molecular patterns, inducing assembly of inflammasomes, activation of caspase-1, and secretion of the pro-inflammatory cytokine IL-1 $\beta$, as well as playing a part in the consequent innate immune response (Gross et al., 2011). In the present study, we established a significant association between the rs 10754558 polymorphism and T2MD, suggesting that mutations in NLRP3 are involved in susceptibility to this disease.

It is well known that insulin resistance is the principal mechanism leading to the development of T2DM, and many inflammatory cytokines, such as IL-1 $\beta$, play an important role in disrupting insulin signaling (Stienstra et al., 2010). Experimental studies have reported that blocking IL-1 $\beta$ signaling with anakinra results in sustained amelioration of T2DM (Akash et al., 2012). Moreover, the results of other investigations using NLRP3-knockout mice suggest that the NLRP3 inflammasome is associated with development of insulin resistance (Stienstra et al., 2010; Vandanmagsar et al., 2011).

Several previous studies have evaluated the connection between NLRP3 polymorphisms and susceptibility to many immune-mediated inflammatory diseases, including rheumatoid arthritis, Crohn's disease, celiac disease, and type 1 diabetes (Villani et al., 2009; Pontillo et al., 2010; Ben Hamad et al., 2012). Ben Hamad et al. (2012) reported that variations in this gene have no effect on rheumatoid arthritis susceptibility in Tunisian and French populations. However, Villani et al. (2009) identified an association between NLRP3 sequence variants and risk of Crohn's disease, 
while Pontillo et al. (2010) established that they contribute to the development of type 1 diabetes and celiac disease in a pediatric population. In our study, we found that the GG and GC+GG genotypes of the rs10754558 polymorphism were significantly associated with increased risk of T2DM. Only one previous study had reported the role of NLRP3 polymorphisms in the development of T2DM, finding that of the two variants tested, rs10754558 was associated with insulin resistance and increased T2DM risk.

In conclusion, we suggest that the NLRP3 rs10754558 polymorphism contributes to the development of T2DM, but that rs7512998 and rs12137901 do not. Further genetic studies involving large sample-sizes are greatly needed to confirm the association between NLRP3 polymorphisms and T2DM risk.

\section{Conflicts of interest}

The authors declare no conflict of interest.

\section{ACKNOWLEDGMENTS}

We thanks for the great help from nurses who help us to collect blood samples, and the Song Wang, Fang Fang and Xisheng Zheng contribute equally to this study.

\section{REFERENCES}

Ahmadi A, Mobasheri M, Hashemi-Nazari SS, Baradaran A, et al. (2014). Prevalence of hypertension and type 2 diabetes mellitus in patients with colorectal cancer and their median survival time: A cohort study. J. Res. Med. Sci. 19: 850-854.

Akash MS, Shen Q, Rehman K and Chen S (2012). Interleukin-1 receptor antagonist: a new therapy for type 2 diabetes mellitus. J. Pharm. Sci. 101: 1647-1658.

Ben Hamad M, Cornelis F, Marzouk S, Chabchoub G, et al. (2012). Association study of CARD8 (p.C10X) and NLRP3 (p.Q705K) variants with rheumatoid arthritis in French and Tunisian populations. Int. J. Immunogenet. 39: 131-136.

Chanprasertyothin S, Jongjaroenprasert W and Ongphiphadhanakul B (2015). The association of soluble IGF2R and IGF2R gene polymorphism with type 2 diabetes. J. Diabetes Res. 2015: 216383.

Chen H, Wei F, Wang L, Wang Z, et al. (2015). MTHFR gene C677T polymorphism and type 2 diabetic nephropathy in Asian populations: a meta-analysis. Int. J. Clin. Exp. Med. 8: 3662-3670.

Chen L, Pei JH, Kuang J, Chen HM, et al. (2015). Effect of lifestyle intervention in patients with type 2 diabetes: a metaanalysis. Metabolism 64: 338-347.

Expert Committee on the Diagnosis and Classification of Diabetes Mellitus (2003). Report of the Expert Committee on the Diagnosis and Classification of Diabetes Mellitus. Diabetes Care 26: S5-S20.

Golshani H, Haghani K, Dousti M and Bakhtiyari S (2015). Association of TNF- $\alpha 308$ G/A polymorphism with type 2 diabetes: A case-control study in the Iranian Kurdish ethnic group. Osong Public Health Res. Perspect. 6: 94-99.

Gross O, Thomas CJ, Guarda G and Tschopp J (2011). The inflammasome: an integrated view. Immunol. Rev. 243: 136-151.

Jiang F, Li S, Pan L and Jia C (2015). Association of the G1057D polymorphism in insulin receptor substrate 2 gene with type 2 diabetes mellitus: a meta-analysis. J. Diabetes Complications 29: 731-736.

Kastbom A, Verma D, Eriksson P, Skogh T, et al. (2008). Genetic variation in proteins of the cryopyrin inflammasome influences susceptibility and severity of rheumatoid arthritis (the Swedish TIRA project). Rheumatology 47: 415-417.

Nanfa D, Sobngwi E, Atogho-Tiedeu B, Noubiap JJ, et al. (2015). Association between the TCF7L2 rs12255372 (G/T) gene polymorphism and type 2 diabetes mellitus in a Cameroonian population: a pilot study. Clin. Transl. Med. 4: 17.

Pontillo A, Brandao L, Guimaraes R, Segat L, et al. (2010). Two SNPs in NLRP3 gene are involved in the predisposition to type-1 diabetes and celiac disease in a pediatric population from northeast Brazil. Autoimmunity 43: 583-589.

Stienstra R, Joosten LA, Koenen T, van Tits B, et al. (2010). The inflammasome-mediated caspase-1 activation controls adipocyte differentiation and insulin sensitivity. Cell Metab. 12: 593-605.

Vandanmagsar B, Youm YH, Ravussin A, Galgani JE, et al. (2011). The NLRP3 inflammasome instigates obesity-induced inflammation and insulin resistance. Nat. Med. 17: 179-188. 
Villani AC, Lemire M, Fortin G, Louis E, et al. (2009). Common variants in the NLRP3 region contribute to Crohn's disease susceptibility. Nat. Genet. 41: 71-76.

Zheng Y, Zhang D, Zhang L, Fu M, et al. (2013). Variants of NLRP3 gene are associated with insulin resistance in Chinese Han population with type-2 diabetes. Gene 530: 151-154. 